\title{
Why should we be concerned about early menarche?
}

\author{
Hae Sang Lee, MD, PhD \\ Department of Pediatrics, Ajou University Hospital, Ajou University School of Medicine, Suwon, Korea
}

\begin{abstract}
Key message
Early menarche is associated with several health problems in later life.

- Early menarche can be caused by environmental factors such as increased subcutaneous fat, a high body mass index, and sugar-sweetened beverages as well as genetic factors.

Health education can prevent early menarche by aiming to reduce the consumption of fructose, high concentrations of which are present in sugar-sweetened beverages.
\end{abstract}

Puberty is a transitional period between childhood and adulthood during which sexual maturation occurs. Menarche, the first menstruation, is a milestone event of pubertal development in girls since it represents the onset of the female reproductive capacity. Menarche, a late event during puberty, usually occurs 2-2.5 years after pubertal onset and is characterized by breast enlargement and pubic hair development. Several studies have reported that the mean age at menarche decreased from 17 years in 1840 to approximately 12 years in 2000 in most developed countries. According to a recent review, the age at menarche has remained stable over the past few decades. ${ }^{1)}$ Age at menarche varies among countries, generations, and races. According to the Korean National Health and Nutrition Survey, in Korea, mean age at menarche decreased from 16.90 years for women born between 1920 and 1925 to 13.79 years for those born between 1980 and $1985^{2}{ }^{2}$

Menarche is a complex phenomenon that is influenced by genetic and environmental factors. Approximately half of the variations in menarcheal age among developing countries may be due to genetic and heritable factors. The age at menarche is similar between mother and daughter, which supports the genetic influence on menarcheal age. Genome-wide association studies reported that several genes or loci, including $L I N 28 B$, TAC3R, ESR1, and 9q31.2, were associated with age at menarche. ${ }^{3)}$ Environmental factors including nutrition, psychosocial variables, and endocrine-disruptor chemicals also influence the timing of menarche. The decrease in menarcheal age can be explained by environmental factors such as nutritional status and the increased prevalence of obesity, although genetic factors also play a crucial role in pubertal progression.

Early menarche is usually defined as menarche before the age of 12 years, although some researchers set it at $\leq 10$ or 11 years. In Korea, $21.4 \%$ of subjects experienced menarche before the age of 12 years in 2001 versus $34.6 \%$ in 2010/2011. ${ }^{4}$ Nutritional habits such as formula feeding during early infancy, increased subcutaneous fat, a high body mass index, early exposure to endocrine-disruptor chemicals, and the consumption of sugarsweetened beverages (SSB) as well as the increased prevalence of precocious puberty are possible factors responsible for early menarche.

Early menarche is associated with physical and psychosocial problems such as anxiety/depression, substance use, and suicidal behavior in adolescents. It can also cause early fusion of the epiphyseal growth plates; therefore, the young woman's final adult height may be shorter than her potential genetic height. In the Korean population, menarche before 12 years of age is associated with an increased risk of metabolic syndrome and type 2 diabetes mellitus in young and middle-aged women. ${ }^{5)}$ It has also been linked to an increased prevalence of obesity, insulin resistance, and hypercholesterolemia in adulthood, culminating in higher risks of cardiovascular diseases such as hypertension, coronary heart disease, stroke, and diabetes in women. Furthermore, women who experience menarche before 12 years of age have a $23 \%$ higher risk of developing breast cancer than those who first menstruate at 15 years of age or later. ${ }^{6}$

Recent public concern about early menarche or precocious puberty has increased due to its adverse consequences in later life. Kim and $\mathrm{Lim}^{7}$ suggested that such consequences can be prevented through health education targeting a reduction in the consumption of fructose, concentrations of which are high in SSB. Carwile et al. ${ }^{8)}$ reported that girls consuming more than 1.5 SSB per day experienced menarche 2.7 months earlier than those consuming less than 2 SSB per week. In Korea, the consumption of SSB rapidly increased from $32 \mathrm{kcal} /$ day to 82 $\mathrm{kcal} / \mathrm{day}$ over an 11-year period.9) The removal of SSB from vending machines in schools and education to encourage the consumption of a balanced diet could be effective ways to reduce fructose consumption.

Corresponding author: Hae Sang Lee MD, PhD. Department of Pediatrics, Ajou University Hospital, Ajou University School of Medicine, 164 World cup-ro, Yeongtonggu, Suwon 16499, Korea 
Menarche is not just a moment during pubertal progression. Its timing, especially when early, may be associated with several health problems in adolescence and adulthood. This finding highlights the need for increased awareness of and efforts to prevent early menarche.

\section{Conflicts of interest}

No potential conflict of interest relevant to this article was reported.

See the article "Early menarche and its consequence in Korean female: reducing fructose intake could be one solution" via https://doi.org/10.3345/cep.2019.00353.

\section{References}

1. Papadimitriou A. The evolution of the age at menarche from prehistorical to modern times. J Pediatr Adolesc Gynecol 2016;29:527-30.

2. Cho GJ, Park HT, Shin JH, Hur JY, Kim YT, Kim SH, et al. Age at menarche in a Korean population: secular trends and influencing factors. Eur J Pediatr 2010;169:89-94.
3. Elks CE, Perry JR, Sulem P, Chasman DI, Franceschini N, He C, et al. Thirty new loci for age at menarche identified by a meta-analysis of genome-wide association studies. Nat Genet 2010;42:1077-85.

4. Lee MH, Kim SH, Oh M, Lee KW, Park MJ. Age at menarche in Korean adolescents: trends and influencing factors. Reprod Health 2016;13:121.

5. Lim JS, Lee HS, Kim EY, Yi KH, Hwang JS. Early menarche increases the risk of Type 2 diabetes in young and middle-aged Korean women. Diabet Med 2015;32:521-5.

6. Key TJ, Verkasalo PK, Banks E. Epidemiology of breast cancer. Lancet Oncol 2001;2:133-40.

7. Kim JH, Lim JS. Early menarche and its consequence in Korean female: reducing fructose intake could be one solution. Clin Exp Pediatr 2020 May 14 [Epub]. https://doi.org/10.3345/cep.2019.00353.

8. Carwile JL, Willett WC, Spiegelman D, Hertzmark E, Rich-Edwards J, Frazier AL, et al. Sugar-sweetened beverage consumption and age at menarche in a prospective study of US girls. Hum Reprod 2015;30:67583.

9. Lim H, Lee HJ, Choue R, Wang Y. Trends in fast-food and sugarsweetened beverage consumption and their association with social environmental status in South Korea. J Acad Nutr Diet 2018;118:122836.e1.

How to cite this article: Lee HS. Why should we be concerned about early menarche? Clin Exp Pediatr 2021;64:26-7. https://doi.org/10.3345/cep.2020.00521 